\title{
Parameter non-identifiability of the Gyllenberg-Webb ODE model
}

\author{
Niklas Hartung
}

Received: date / Accepted: date

\begin{abstract}
An ODE model introduced by Gyllenberg and Webb (1989) describes tumour growth in terms of the dynamics between proliferating and quiescent cell states. The passage from one state to another and vice versa is modelled by two functions $r_{o}$ and $r_{i}$ depending on the total tumour size. As these functions do not represent any observable quantities, they have to be identified from the observations.

In this paper we show that there is an infinite number of pairs $\left(r_{o}, r_{i}\right)$ corresponding to the same solution of the ODE system and the functions $\left(r_{o}, r_{i}\right)$ will be classified in terms of this equivalence. Surprisingly, the technique used for this classification permits a uniqueness proof of the solution of the ODE model in a non-Lipschitz case.

The reasoning can be widened to a more general setting including an extension of the Gyllenberg-Webb model with a nonlinear birth rate. The relevance of this result is discussed in a preclinical application scenario.
\end{abstract}

Keywords Tumour Growth · Quiescence · Parameter identifiability

Mathematics Subject Classification (2000) 92C50 - 34A12 34 A55

\section{Introduction}

Cancer is a disease of abnormal tissue growth induced by a series of growthpromoting genetic changes (Hanahan and Weinberg, 2011). It is a leading cause of death worldwide (WHO, 2012). Crucial among the many difficulties in cancer treatment is the existence of a subpopulation of cancer cells inherently more resistant to many types of therapy: the quiescent cells (Alarcon and Jensen, 2011; Hittelman et al, 2010; Mellor et al, 2005). Quiescence is a reversible state

Niklas Hartung

CMI 39 rue Frédéric Joliot-Curie 13453 Marseille cedex 13, France

E-mail: hartung@cmi.univ-mrs.fr 
of nondivision that is functionally different from cell cycle arrest as it can be prolonged and exhibits some characteristic gene expression patterns (Coller et al, 2006; Coller, 2011). Cancerous cells are known to undergo quiescence under environmental stress like hypoxia and glucose deficiency, which is commonly the case due to poor and aberrant tumour vascularisation, or during therapy application (Gardner et al, 2001; Hanahan and Weinberg, 2011; Liu et al, 2011). When environmental conditions are favourable, quiescent cancer cells can re-enter the cell cycle. In this way, quiescence contributes to therapy failure and recurrence of the disease.

As tumour cells are principally characterised by their rapid and ongoing division, many anticancer agents operate on dividing cells, either specifically during DNA synthesis and mitosis or in a phase-nonspecific manner (e.g. radiotherapy). Naturally, phase-specific anticancer drugs have no effect on noncycling cells. Beyond that, the tumour microenvironment, and thus quiescence induced by environmental stress, has shown to be associated with resistance to phase-nonspecific agents, including radiotherapy, chemotherapy and gene therapy (Redmond et al, 2008; Tredan et al, 2007; Mellor et al, 2005; Wallbaum et al, 2009). Strategies to circumvent this resistance by reactivating quiescent cells are an active field of current research (Kyle et al, 2012).

Tumour cell proliferation measures by immunohistochemical techniques, notably the Ki67 antibody MIB-1, are well established (Brown and Gatter, 2002; Colozza et al, 2005). More recently, an in vivo following of cell proliferation in murine models has become possible. High-resolution measurements can be obtained in vivo by combining gene therapy and SPECT (single-photon emission computed tomography) imaging (Franken et al, 2010; Richard Fiardo et al, 2012), see Figure 1. These new measurement instruments calls for a review of mathematical models distinguishing between proliferating and quiescent cell states and invite to critically analyse their applicability, ultimately aiming to shed light on the mechanisms inherent in the dynamics between proliferating and quiescent cells that lead to tumour progression or tumour regression under different therapeutic regimes. We will focus on models relevant for SPECT imaging of proliferating cells.

The usage of mathematical growth models has yielded important contributions to solid tumour growth research in the last decades (Araujo and McElwain, 2004). Amongst the many approaches, minimally parametrised models, aiming at reproducing the observed phenomena in a previously defined setting without integrating all the underlying biological mechanisms explicitly, play a prominent role. The main interest of such models is that the small number of parameters included in such models results in less complex numerical simulations and an easier parameter identification.

The oldest and perhaps most cited phenomenological tumour growth model is the Gompertzian growth model, exhibiting an exponential increase of tumour volume at early stages and a subsequent deceleration with tumour volume converging to a maximal value, the carrying capacity (Laird, 1964). The Gompertz model has also been integrated in more complex tumour growth models (Iwata et al, 2000; Hahnfeldt et al, 1999). However, there is nothing unique about the 
Gompertz model. As was already pointed out by Feller (1939), different models having such S-shaped (sigmoidal) solutions can fit data equally well. See for example Hart et al (1998) and Simeoni et al (2004) for some alternative phenomenological models used to describe tumour growth.

Numerous models distinguish between proliferating and quiescent cells (and possibly other effects) (Spinelli et al, 2006; Smallbone et al, 2008; Ubezio and Cameron, 2008), among them a model established by Gyllenberg and Webb (1989) (in the following referred to as the Gyllenberg-Webb model). This model is based on the coupled dynamics between proliferating and quiescent cells, realised by two passage rates, from proliferating to quiescent and vice versa. For the modelling of quiescence, the Gyllenberg-Webb model is exemplary as it is a minimal approach while having mechanistic components. Amongst others, D'Onofrio et al (2011) have studied this model, looking for compatibility with certain scalar ODE models. This point will be further elaborated in Section 3. In view of the precise in vivo imaging techniques of proliferating cells available, the call for an applicability study of the Gyllenberg-Webb model arises naturally, one of its centrepieces being a parameter identifiability study.

In the following section, the model established by Gyllenberg and Webb will be presented and their mathematical analysis will briefly be recapitulated. Section 3 covers the parameter identifiability problem for the Gyllenberg-Webb model. In particular, an explicit equivalence relation will be established that groups the passage rates corresponding to the same solutions of the ODEs. Interestingly, this approach will permit a uniqueness proof of these solutions for non-Lipschitz passage rates. In Section 4, the reasoning will be generalised to a larger class of ODEs containing an extended form of the Gyllenberg-Webb model with a nonlinear birth rate. Finally, the consequences for the applicability of the Gyllenberg-Webb model is discussed.

\section{The Gyllenberg-Webb model}

The generic ODE model proposed by Gyllenberg and Webb (1989) distinguishes between proliferating $(P)$ and quiescent $(Q)$ cells and is based on a dynamic state change between these two classes. Proliferating cells reproduce and die with a specific growth rate (birth rate minus death rate) $b>0$ and can become quiescent. Quiescent cells cannot reproduce but can become proliferating again. Death of quiescent cells is described by a mortality parameter $\mu \geqslant 0$. The total tumour size $N$ is the sum of proliferating and quiescent cells. Thus, the model is given by the following system of ordinary differential equations (ODE):

$$
\begin{aligned}
& P^{\prime}(t)=\left[b-r_{o}(N(t))\right] P(t)+r_{i}(N(t)) Q(t), \\
& Q^{\prime}(t)=r_{o}(N(t)) P(t)-\left[r_{i}(N(t))+\mu\right] Q(t), \\
& N(t)=P(t)+Q(t), \\
& P(0)=P_{0}>0, \quad Q(0)=Q_{0} \geqslant 0 .
\end{aligned}
$$




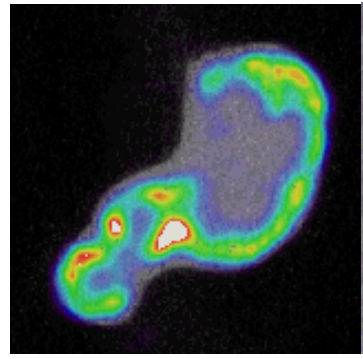

(a)

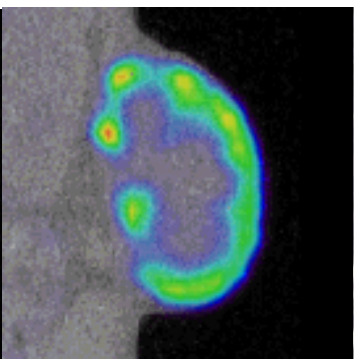

(b)

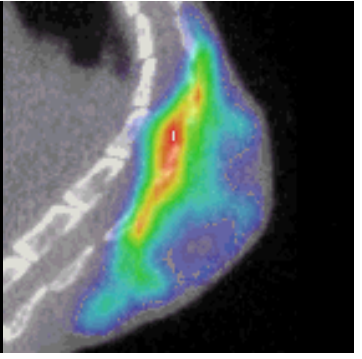

(c)

Fig. 1: SPECT images of colorectal human cancer cells (HT-29) that were modified to express a iodine transportation protein and implanted in an immunodeficient mouse. Iodine 123 has been used as a radiotracer. Images obtained from T. Pourcher of the TIRO laboratory in Nice (France), with permission. In the outer layers of the tumour, an important accumulation of the radiotracer has been registered, which corresponds to the proliferating cancer cells. The radiotracer does not accumulate in quiescent cells and they are therefore indistinguishable from dead cells.

The functions $r_{o}$ and $r_{i}$ describe the passage between the two states and are supposed to depend on the total tumour size. The function $r_{o}$ is assumed continuously increasing and $r_{i}$ is assumed continuously decreasing.

Gyllenberg and Webb's results will be stated in an abbreviated form, and we are going to complement them thereafter. We refer to Gyllenberg and Webb (1989) for their rigorous and detailed discussion.

Proposition 1 (The case $r_{i} \equiv 0$ and $\mu=0$ ) Suppose that $\lim _{N \rightarrow+\infty} r_{o}(N)>$ $b, r_{i} \equiv 0$ and $\mu=0$. Then $N=P+Q$ is increasing and reaches a plateau $N^{*}<+\infty$, which is the unique solution of

$$
b\left(N^{*}-Q_{0}\right)=\int_{N_{0}}^{N^{*}} r_{o}(N) d N .
$$

Furthermore $\lim _{t \rightarrow+\infty} P(t)=0$.

Proposition 2 (The case $r_{i}(N) \geqslant l_{i}>0$ and $\mu=0$ ) Suppose that $r_{i}(N) \geqslant$ $l_{i}>0$ for all $N \geqslant 0$ and $\mu=0$. Then $N$ is increasing and $N^{*}:=\lim _{t \rightarrow+\infty} N(t)=$ $+\infty$. If $r_{o}$ is bounded, then $\lim _{t \rightarrow+\infty} P(t)=+\infty$. If $r_{o}$ is unbounded, then

$$
\begin{aligned}
& \limsup _{t \rightarrow+\infty} P(t) \leqslant \limsup _{N \rightarrow+\infty} \frac{N r_{i}(N)}{r_{o}(N)}, \\
& \liminf _{t \rightarrow+\infty} P(t) \geqslant \liminf _{N \rightarrow+\infty} \frac{N r_{i}(N)}{r_{o}(N)} .
\end{aligned}
$$


Proposition 3 (The case $r_{i}(N) \geqslant l_{i}>0$ and $\mu>0$ ) Suppose $r_{i}(N) \geqslant$ $l_{i}>0$ for all $N \geqslant 0$ and $\mu>0$. If the tendency to become quiescent is sufficiently low in small tumours ( $N$ small) and sufficiently high in large tumours ( $N$ large), then there exists a unique nontrivial equilibrium that is globally asymptotically stable. The total tumour size $N$ tends to the unique solution of

$$
r_{o}\left(N^{*}\right)=b\left(1+\frac{r_{i}\left(N^{*}\right)}{\mu}\right) .
$$

Gyllenberg and Webb remarked that in all of these cases their hypotheses implied a diminishing of the growth fraction $\frac{P}{N}$ and gave two examples of functions $r_{o}$ with $r_{i} \equiv 0$ such that the total tumour size $N$ satisfies the logistic and Gompertz equation, respectively.

\section{Equivalent parameter sets and uniqueness of solutions in the Gyllenberg-Webb model}

If we want to evaluate the quantitative performance of the Gyllenberg-Webb model, the rates $r_{o}$ and $r_{i}$ need to be specified. However, it is difficult to justify a particular choice for these functions as they do not represent any observable quantities. It is therefore interesting to study the identifiability problem of the parameters from given solutions $(P, Q)$ of the ODE system (1-4), which are measurable.

Hypothesis (H) In this section, we will assume that $b>0, \mu=0$, $r_{o}$ is a positive nondecreasing continuous function and that $r_{i}$ is a non-negative nonincreasing continuous function, with $\lim _{N \rightarrow+\infty} r_{o}(N)+$ $r_{i}(N)>b$.

This setting allows an easier presentation of the principal result but it has also a biological significance. In subcutaneous tumours xenografted in mice, an often used experimental model, the dead tissue is often completely surrounded by tumour cells (Ribba et al, 2011). It is therefore reasonable to consider the dead tissue as a contribution to the total tumour volume, which can be done by posing $\mu=0$. If we want the model to reproduce the "recruiting" of quiescent cells during a therapy, we need to consider a passage rate $r_{i}$ which is not identically zero. Note that $r_{i}$ is allowed to tend to zero at infinity and there can even exist $N_{L}>0$ such that $r_{i}\left(N_{L}\right)=0$ (and thus $\left.r_{i}\right|_{\left[N_{L},+\infty\right)} \equiv 0$ ), a case that was not included in the analysis of Gyllenberg and Webb. As will be shown in this section, any reasonable ODE model for tumour growth depending only on total tumour size, like the Gompertzian and the logistic growth model, can be reproduced by a variety of parameter sets $\left(b, r_{o}, r_{i}\right)$, with $r_{i}$ non identically zero but vanishing in $N_{L}>0$. The case $\mu>0$ will be covered in Section 4 . 
3.1 Direct problem

Before analysing the identifiability problem let us state two results on the direct problem for the sake of completeness:

Proposition 4 (Global existence and positivity of solutions of (1-4)) Let $P_{0}>0, Q_{0} \geqslant 0, b>0, \mu \geqslant 0, r_{o}$ continuous, nondecreasing and positive, $r_{i}$ continuous, nonincreasing and non-negative. Then there is at least one solution $(P, Q)$ of (1-4), defined for all times $t \in[0,+\infty)$. All solutions are positive for $t \in(0,+\infty)$.

Proof A detailed proof can be found in Hartung (2011). Positivity can be established with standard manipulations of equations (1-4) and global existence uses the explosion theorem for finite times of existence (Amann and Escher, 2006).

The asymptotics of $N$ and $P$ have been studied by Gyllenberg and Webb in different scenarios, including the one specified by the hypotheses $(\mathrm{H})$ with a technical restriction $r_{i} \geqslant C>0$. We will propose a different proof that is valid for $\lim _{N \rightarrow+\infty} r_{i}(N)=0$ as well and in which the line of argument is much simpler.

Proposition 5 (Asymptotics) Let us suppose that hypothesis (H) holds and let us note

$$
N_{L}:=\inf \left\{N>0: r_{i}(N)=0\right\}, N^{*}:=\lim _{t \rightarrow+\infty} N(t) .
$$

Then we have the following:

$$
\begin{gathered}
N_{L}=+\infty \Rightarrow N^{*}=+\infty, N_{L}<+\infty \Rightarrow N^{*}<+\infty, \\
\liminf _{t \rightarrow \infty} P(t) \geqslant \liminf _{N \rightarrow \infty} \frac{r_{i}(N) N}{r_{o}(N)+r_{i}(N)-b}, \\
\limsup _{t \rightarrow \infty} P(t) \leqslant \limsup _{N \rightarrow \infty} \frac{r_{i}(N) N}{r_{o}(N)+r_{i}(N)-b} .
\end{gathered}
$$

Proof The asymptotic behaviour of $N$ can be shown with standard techniques (see Hartung, 2011; Gyllenberg and Webb, 1989, for further details). Let us address the asymptotic behaviour of $P$. For the limsup estimation let us consider a sequence $t_{k}$ that tends to infinity such that

$$
\lim _{k \rightarrow+\infty} P\left(t_{k}\right)=\limsup _{t \rightarrow+\infty} P(t), \liminf _{k \rightarrow+\infty} P^{\prime}\left(t_{k}\right) \in[0,+\infty] .
$$

Such a sequence exists for any inferiorly bounded differentiable function. We still denote by $t_{k}$ a subsequence for which $\lim _{k \rightarrow+\infty} P^{\prime}\left(t_{k}\right) \in[0,+\infty]$ exists and we rewrite (1) replacing $Q$ by $N-P$ :

$$
\frac{N\left(t_{k}\right) r_{i}\left(N\left(t_{k}\right)\right)}{r_{o}\left(N\left(t_{k}\right)\right)+r_{i}\left(N\left(t_{k}\right)\right)-b}=P\left(t_{k}\right)+P^{\prime}\left(t_{k}\right) V\left(t_{k}\right),
$$


with $V\left(t_{k}\right):=\frac{1}{r_{o}\left(N\left(t_{k}\right)\right)+r_{i}\left(N\left(t_{k}\right)\right)-b}$ and $k$ sufficiently large such that $r_{o}\left(N\left(t_{l}\right)+\right.$ $r_{i}\left(N\left(t_{l}\right)\right)>b$ for all $l \geqslant k$. We remark that $r_{o}\left(N\left(t_{l}\right)+r_{i}\left(N\left(t_{l}\right)\right)-b\right.$ is uniformly positive by hypothesis $(\mathrm{H})$, and up to passing to a maximising subsequence (still denoted $t_{k}$ ) of $V\left(t_{k}\right)$ we can assume that $\lim _{k \rightarrow+\infty} V\left(t_{k}\right) \in[0,+\infty)$ exists.

We pass to the limit $k \rightarrow+\infty$ :

$$
\lim _{k \rightarrow+\infty} \frac{N\left(t_{k}\right) r_{i}\left(N\left(t_{k}\right)\right)}{r_{o}\left(N\left(t_{k}\right)\right)+r_{i}\left(N\left(t_{k}\right)\right)-b}=\limsup _{t \rightarrow+\infty} P(t)+\lim _{k \rightarrow+\infty} P^{\prime}\left(t_{k}\right) \lim _{k \rightarrow+\infty} V\left(t_{k}\right)
$$

The last term is non-negative by construction of $t_{k}$. Furthermore,

$$
\limsup _{N \rightarrow+\infty} \frac{N r_{i}(N)}{r_{o}(N)+r_{i}(N)-b} \geqslant \lim _{k \rightarrow+\infty} \frac{N\left(t_{k}\right) r_{i}\left(N\left(t_{k}\right)\right)}{r_{o}\left(N\left(t_{k}\right)\right)+r_{i}\left(N\left(t_{k}\right)\right)-b} .
$$

This yields (6). Similarly, inequality (5) can be obtained with a minimising sequence satisfying $\limsup P^{\prime}\left(t_{k}\right) \leqslant 0$ (considering separately the case $\left.\liminf _{t \rightarrow+\infty} P(t)=+\infty\right)$.

\subsection{Main result}

The analysis in this section is founded on the following basic consideration: As we assume that $\mu=0$, equations (1-2) imply $N^{\prime}=b P$ hence $N^{\prime \prime}=b P^{\prime}$. By inserting these two equations in (1), one obtains the second order equation

$$
N^{\prime \prime}=\left(b-r_{o}(N)-r_{i}(N)\right) N^{\prime}+b N r_{i}(N) .
$$

We will now impose a first order ODE growth $N^{\prime}=f(N)$ on the total tumour size $N=P+Q$ to gain insight on the nature of the passage rates $r_{o}$ and $r_{i}$. At this point, it may seem unreasonable to add a constraint to the Gyllenberg-Webb model, but we will see that a large variety of passage rates can be characterised in this way. The constraint can be thought of as an empirical macroscopic growth model fitting data from preclinical or clinical observations.

In the case of the Gompertz growth model, this question has been studied by Kozusko and Bajzer (2003). Moreover, D'Onofrio, Fasano, and Monechi (2011) have introduced a notion of compatibility essentially equivalent to the following definition. The present study is yet not redundant as the objectives are different: The mentioned articles aimed at the fusion of certain ODE models with the Gyllenberg-Webb model, whereas the present study is focussed on the applicability of the Gyllenberg-Webb model, a question not addressed in the previous studies. The following sections and especially the discussion will further clarify this important difference.

By inserting $N^{\prime}=f(N)$ in (7), one obtains

$$
f^{\prime}(N) f(N)=\left(b-r_{o}(N)-r_{i}(N)\right) f(N)+b r_{i}(N) N,
$$

which motivates the following definition: 
Definition 1 (Compatibility equation)

For $f \in C^{1}\left(\left(N_{0}, N^{*}\right), \mathbb{R}^{+}\right) \cap C^{0}\left(\left[N_{0}, N^{*}\right), \mathbb{R}^{+}\right)$, we call

$$
f^{\prime}(n) f(n)=\left(b-r_{o}(n)-r_{i}(n)\right) f(n)+b r_{i}(n) n,
$$

compatibility equation and we say that the parameters $\left(b, r_{o}, r_{i}\right)$ are compatible with the function $f$ if $(8)$ holds on $\left(N_{0}, N^{*}\right)$ and if $f\left(N_{0}\right)=b P_{0}$.

With this definition, we can now state the main result.

Theorem 1 Let $b>0, r_{o}$ continuous and increasing, $r_{i}$ continuous and decreasing.

- There is a unique solution $(P, Q)$ of the Gyllenberg-Webb model (1-4)

- There is a unique function $f_{\left(b, r_{o}, r_{i}\right)} \in C^{1}\left(\left[N_{0}, N^{*}\right), \mathbb{R}^{+}\right)$that satisfies the compatibility equation (8) and the condition $f_{\left(b, r_{o}, r_{i}\right)}\left(N_{0}\right)=b P_{0}$. We will call $f_{\left(b, r_{o}, r_{i}\right)}$ the associated function.

- $N=P+Q$ is the unique solution of $N^{\prime}(t)=f_{\left(b, r_{o}, r_{i}\right)}(N(t))$.

Remark 1 Observe that the proof technique also yields uniqueness of the solutions $P$ and $Q$ of (1-4) when $r_{o}$ and $r_{i}$ are not supposed locally Lipschitz continuous.

Proof (Proof of Theorem 1.)

- Associated function.

Let us start by considering equation (8). With the change of variables $g=f^{2}$ and by writing $a_{1}(n)=2\left(b-r_{o}(n)-r_{i}(n)\right)$ and $a_{2}(n)=2 b n r_{i}(n)$, we search the solution $g$ of

$$
\begin{aligned}
g^{\prime}(n) & =a_{1}(n) \sqrt{g(n)}+a_{2}(n), \\
g\left(N_{0}\right) & =\left(b P_{0}\right)^{2},
\end{aligned}
$$

the existence and uniqueness of which is assured by the Picard-Lindelöf theorem as long as $g$ does not vanish. Let $\tilde{N}=\sup \left\{n \geqslant N_{0}: g>0\right.$ on $\left.\left(N_{0}, n\right)\right\}$. Thus, on $\left(N_{0}, \tilde{N}\right)$ the function $f_{\left(b, r_{o}, r_{i}\right)}:=\sqrt{g}$ is well defined and the property

$$
\tilde{N}<+\infty \Rightarrow \lim _{n \rightarrow \tilde{N}} f(n)=0
$$

follows as a direct consequence of the definition of $\tilde{N}$ using the fact that $g$ is continuous. Note that this result could be established for $f$ directly, but it is the upper change of variables which will be needed for the generalisation in Section 4.

$-N$ is solution of the $O D E$.

Let $(P, Q)$ be a solution of (1-4). Global existence and positivity have been established in Proposition 4. Recall that $N=P+Q$ satisfies (7) and that $N^{*}:=\lim _{t \rightarrow+\infty} N(t)$. As $N^{\prime}=b P>0$, we can define $h(n):=N^{\prime}\left(N^{-1}(n)\right)$ on $\left[N_{0}, N^{*}\right)$. We have $h(N(t))=N^{\prime}(t)$ and $h^{\prime}(N(t)) h(N(t))=N^{\prime \prime}(t)$. Thus, on $\left(N_{0}, N^{*}\right), h$ is a solution of $(8)$, hence $h \equiv f_{\left(b, r_{o}, r_{i}\right)}$ on $\left(N_{0}, \min \left(\tilde{N}, N^{*}\right)\right)$. 
- $\tilde{N}=N^{*}$ holds.

We have $f_{\left(b, r_{o}, r_{i}\right)}(N(t))=b P(t)>0$ for all $t>0$, hence $\tilde{N} \geqslant N^{*}$. On the other hand, if $N^{*}<+\infty$,

$$
f_{\left(b, r_{o}, r_{i}\right)}\left(N^{*}\right)=\lim _{t \rightarrow+\infty} f_{\left(b, r_{o}, r_{i}\right)}(N(t))=\lim _{t \rightarrow+\infty} N^{\prime}(t)=0
$$

since $N^{\prime}=b P>0$, hence $\tilde{N}=N^{*}$.

- Uniqueness of solutions $(P, Q)$.

For every solution $N=P+Q$, we have $N^{\prime}(t)=f_{\left(b, r_{o}, r_{i}\right)}(N(t))$ on $[0,+\infty)$. As $f_{\left(b, r_{o}, r_{i}\right)}$ is locally Lipschitz, $N$ can be determined unambiguously from $f_{\left(b, r_{o}, r_{i}\right)}$. Finally, $P=\frac{N^{\prime}}{b}$ and $Q=N-P$ are determined unambiguously from $N$.

Theorem 1 allows for an explicit characterisation of the parameters corresponding to the same solutions of the ODE:

Definition 2 (Parameter equivalence) For given $b>0, P_{0}$ and $Q_{0}$, we can define an equivalence relation in the set $\left\{\left(r_{o}, r_{i}\right) \in C^{0}\left(\left(N_{0},+\infty\right)\right)^{2}: r_{o}\right.$ increasing and $r_{i}$ decreasing $\}$ :

$$
\left(r_{o}^{1}, r_{i}^{1}\right) \sim\left(r_{o}^{2}, r_{i}^{2}\right) \Leftrightarrow N_{1}^{*}=N_{2}^{*} \text { and } f_{\left(b, r_{o}^{1}, r_{i}^{1}\right)} \equiv f_{\left(b, r_{o}^{2}, r_{i}^{2}\right)},
$$

where the functions on the right-hand side are the functions associated to $\left(b, r_{o}^{1}, r_{i}^{1}\right)$ and $\left(b, r_{o}^{2}, r_{i}^{2}\right)$, respectively, as defined in the preceding theorem. This proves their existence and uniqueness, as well as the fact that the solutions $(P, Q)$ coincide if and only if they are in the same equivalence class.

\subsection{Consequences}

Corollary 1 (Non-identifiability and descriptive flexibility) Let $P_{0}>$ $0, Q_{0} \geqslant 0$ and $N_{0}=P_{0}+Q_{0}$. Suppose that $f \in C^{1}\left(\left(N_{0}, N^{*}\right), \mathbb{R}^{+}\right)$with $N^{*}=\infty$ or $\lim _{N \rightarrow N^{*}} f(N)=0$. Then there are $b>0$ and infinitely many passage rates $\left(r_{o}, r_{i}\right)$ continuous such that the associated solutions of the Gyllenberg-Webb model $(P, Q)$ coincide and such that the total tumour size $N=P+Q$ is the solution of the corresponding ODE model $N^{\prime}=f(N), N(0)=N_{0}$. If $f^{\prime}$ is decreasing (e.g. Gompertz model, logistic model, etc.), there exist infinitely many couples $\left(r_{o}, r_{i}\right)$ with $r_{o}$ increasing and $r_{i}$ decreasing.

Proof Define $b>0$ by the relation $f\left(N_{0}\right)=b P_{0}$ and let $r_{i}$ be any nonnegative continuous function. Then we solve the compatibility equation (8) for $r_{o}$ such that $f \equiv f_{\left(b, r_{o}, r_{i}\right)}$ (that is, $r_{o}=b-f^{\prime}+r_{i}(b N / f-1)$ ). Every parameter set $\left(r_{o}, r_{i}\right)$ constructed in this manner lies in the same equivalence class, and Theorem 1 yields the result.

If $f^{\prime}$ is decreasing, the preceding construction with $r_{i} \equiv 0$ yields an increasing function $r_{o}$. It can now easily be seen that if $r_{i}$ is small enough (and decreasing), the corresponding function $r_{o}$ is still increasing. 
Remark 2 In the previous proof, monotony was shown for small perturbations of $r_{i} \equiv 0$. In the following corollary, this is shown for arbitrarily big perturbations in a special case.

Corollary 2 (Parameter estimation with four unknown parameters.) Let $(P, Q)$ be a solution of (1-4) such that the total tumour size $N=P+Q$ follows the logistic equation $N^{\prime}=f(N)=\kappa N(1-N / \theta)$ with $\kappa, \theta>0$ and $b>0$ such that $f\left(N_{0}\right)=b P_{0}$.

Then the parameter estimation problem

Calculate $\alpha>0, \beta>0, \gamma>0, \delta>0$ such that the solutions $\left(P_{\alpha, \beta, \gamma, \delta}, Q_{\alpha, \beta, \gamma, \delta}\right)$ of (1-4) associated to the functions $r_{o}(n)=\alpha+\beta n$ and $r_{i}(n)=(\gamma-\delta n)^{+}$minimise the functional

$$
J(\alpha, \beta, \gamma, \delta):=\left\|\left(P_{\alpha, \beta, \gamma, \delta}, Q_{\alpha, \beta, \gamma, \delta}\right)-(P, Q)\right\|
$$

is ill-posed.

Proof The equivalent parameters are given by

$$
\kappa\left(1-\frac{2 n}{\theta}\right)=b-r_{o}(n)-\left(\frac{b}{\kappa(1-n / \theta)}-1\right) r_{i}(n) .
$$

Hence, for all $s>0$, the functions $r_{o}^{s}(n)=(1+s)(\kappa-b)+\frac{(2+s) \kappa}{\theta} n$ and $r_{i}^{s}(n)=s \kappa\left(1-\frac{n}{\theta}\right)$ are equivalent with associated function $f(n)=\kappa n(1-n / \theta)$ (cf. Figure 2).

Remark 3 In the previous example, the total tumour size $N$ followed the logistic curve. The same reasoning can be used for other sigmoidal growth curves, but the parametrisation of $r_{o}$ and $r_{i}$ becomes more complicated in this case.

\section{A generalisation of Theorem 1}

To a certain extent, the methods used in the preceding section can be generalised.

\subsection{Statement}

Consider the following system of ODEs:

$$
\left\{\begin{array}{l}
x^{\prime}=F_{1}(x, y) \\
y^{\prime}=F_{2}(x, y)
\end{array}\right.
$$

along with the initial conditions $x(0)=x_{0}>0, y(0)=y_{0}>0$.

Theorem 2 Let $F_{1} \in C^{0}\left(\mathbb{R}^{+} \times \mathbb{R}^{+}\right)$locally Lipschitz with respect to $x$ and $F_{2} \in C^{2}\left(\mathbb{R}^{+} \times \mathbb{R}^{+}\right)$. Suppose that $F_{2}>0$ and that there is a function $\Phi \in$ $C^{0,1}\left(\mathbb{R}^{+} \times \mathbb{R}^{+}\right)$such that any solution $(x, y)$ of $(9)$ satisfies $x=\Phi\left(y, y^{\prime}\right)$. Then there is exactly one local solution of (9). 


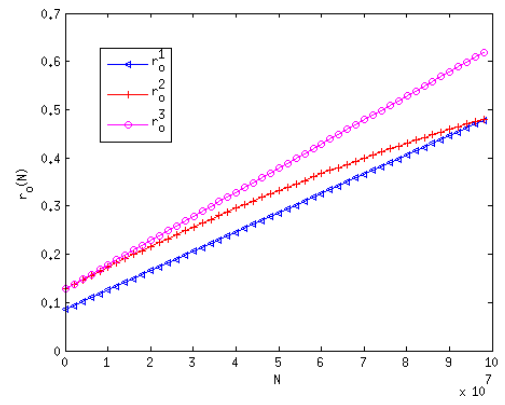

(a) Passage rates $r_{o}$

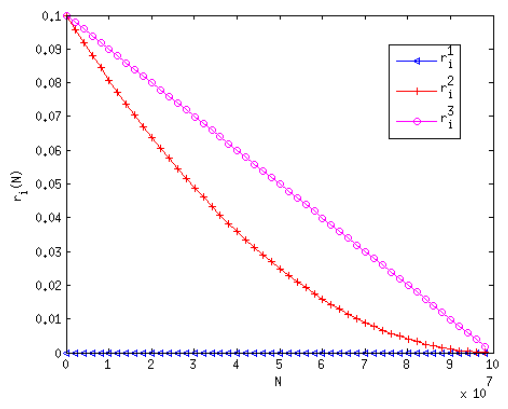

(b) Passage rates $r_{i}$

Fig. 2: An example of equivalent passage rates: the three pairs $\left(r_{o}, r_{i}\right)$ are compatible with the same logistic growth model imposed on the total tumour size. Thus, the solutions $(P, Q)$ of (1-4) coincide. Passage rates: $r_{o}^{1}(N)=$ $0.0854+4 \cdot 10^{-9} N, r_{o}^{2}(N)=0.128+4.573 \cdot 10^{-9} N-10^{-17} N^{2}, r_{o}^{3}(N)=$ $0.128+5 \cdot 10^{-9} N, r_{i}^{1} \equiv 0, r_{i}^{2}(N)=10^{-17}\left(N-10^{8}\right)$ and $r_{i}^{3}(N)=0.1-10^{-9} N$. The passage rates $r_{o}^{j}$ have been calculated from $r_{i}^{j}, j=1,2,3$ and the logistic growth function $f(N)=0.2 N\left(1-N / 10^{8}\right)$ via relation (8). Initial values: $P_{0}=7 \cdot 10^{4}, Q_{0}=3 \cdot 10^{4}$.

Proof Let $(x, y)$ be a solution of (9). By virtue of our hypotheses, there is a function $\Phi \in C^{0,1}\left(\mathbb{R}^{2}\right)$ such that $x=\Phi\left(y, y^{\prime}\right)$. Thus,

$$
y^{\prime \prime}=\left(\frac{d F_{2}}{d x} F_{1}+\frac{d F_{2}}{d y} F_{2}\right)\left(\Phi\left(y, y^{\prime}\right), y\right) .
$$

As $y^{\prime}=F_{2}(x, y)>0, y$ is invertible. Let $f:=y^{\prime}\left(y^{-1}\right) \in C^{1}\left(\mathbb{R}^{+}\right)$. We replace the derivatives $y^{\prime}$ and $y^{\prime \prime}$ by $f(y)$ and $f^{\prime}(y) f(y)$, respectively, and pose $g=f^{2}$. Thus, (10) becomes

$$
g^{\prime}(y)=2\left(\frac{d F_{2}}{d x} F_{1}+\frac{d F_{2}}{d y} F_{2}\right)(\Phi(y, \sqrt{g(y)}), y),
$$

where $y=y(t)$. Note that (11) is an autonomous equation. As the function on the right-hand side is locally Lipschitz in $g(y)$ as long as $g$ does not vanish, the problem

$$
(\tilde{P})\left\{\begin{array}{c}
g^{\prime}(s)=2\left(\frac{d F_{2}}{d x} F_{1}+\frac{d F_{2}}{d y} F_{2}\right)(\Phi(s, \sqrt{g(s)}), s), \\
g\left(y_{0}\right)=F_{2}\left(x_{0}, y_{0}\right)>0
\end{array}\right.
$$

has a unique solution $\tilde{g} \in C^{1}\left(\left(y_{0}, y^{*}\right), \mathbb{R}^{+}\right)$where $y^{*}=+\infty$ or $g\left(y^{*}\right)=0$. Let us note $\tilde{f}=\sqrt{\tilde{g}}$. As $(11)$ holds for $f=y^{\prime}\left(y^{-1}\right)$ on $\left(y_{0}, \lim _{t \rightarrow+\infty} y(t)\right)$, we have $f \equiv \tilde{f}$ and $y^{*}=\lim _{t \rightarrow+\infty} y(t)$. Thus, $y$ can be determined from $\tilde{g}$ unambiguously. Using $x=\Phi\left(y, y^{\prime}\right)$ we conclude that the solution of (9) is unique. 
Remark 4 Global existence needs additional hypotheses on $F_{1}$ and $F_{2}$. This issue is not dealt with in the present work, as it is, first of all, disconnected from the proof technique resulting from the parameter identification problem and second easy to prove in the framework of the following applications.

As can be seen in (12), the following generalisation of (8) arises naturally from the proof:

Definition 3 Suppose that $F_{1}$ and $F_{2}$ depend on some parameters (real numbers or functions) $\Psi=\left(\psi_{i}\right)_{i=1}^{M}$. We call

$$
f^{\prime}(s) f(s)=\left(\frac{d F_{2}}{d x} F_{1}+\frac{d F_{2}}{d y} F_{2}\right)(\Phi(s, f(s)), s)
$$

a generalised compatibility equation and by means of Theorem 2 , this equation defines an equivalence relation in the set of the admissible parameters $\Psi$.

\subsection{Applications of Theorem 2}

Gyllenberg-Webb model, $\mu>0$ :

We have

$$
\begin{aligned}
P^{\prime} & =\left[b-r_{o}(N)-r_{i}(N)\right] P+r_{i}(N) N, \\
N^{\prime} & =(b+\mu) P-\mu N .
\end{aligned}
$$

Thus, $F_{1}(P, N)=\left[b-r_{o}(N)-r_{i}(N)\right] P+r_{i}(N) N, F_{2}(P, N)=(b+\mu) P-\mu N$, $\Phi\left(N, N^{\prime}\right)=\frac{N^{\prime}+\mu N}{b+\mu}$. Note that $F_{2}(P, N)>0$ is not true for all $P>0, N>0$, but the uniqueness result applies if every solution satisfies $F_{2}(P(t), N(t))>$ $0 \forall t \geqslant 0$. In their article, Gyllenberg and Webb presented cases where the total tumour size $N$ was not monotone, so we cannot hope to establish $F_{2}(P(t), N(t))>0$ for an arbitrary parameter set. However, starting from a positive function $f \in C^{1}\left(N_{0}, N^{*}\right)$, the generalised equivalence relation allows us to specify which parameters are associated to $N$ satisfying $N^{\prime}=f(N)$. Let us be explicit:

$$
\begin{aligned}
f^{\prime}(n) f(n)= & (b+\mu)\left(\left(b-r_{o}(n)-r_{i}(n)\right) \frac{f(n)+\mu n}{b+\mu}+r_{i}(n) n\right) \\
& -\mu\left((b+\mu) \frac{f(n)+\mu n}{b+\mu}-\mu n\right) \\
= & f(n)\left(b-\mu-r_{o}(n)-r_{i}(n)\right)+\left(b \mu-\mu r_{o}(n)+b r_{i}(n)\right) n .
\end{aligned}
$$

\section{Extended Gyllenberg-Webb model:}

The proliferation term $b P$ could be replaced by a nonlinear function $\varphi(P, N)$ in order to account for phenomena like angiogenesis or metastatic emission. For 
example, Ribba et al (2011) used a proliferation term of the form $\varphi(P, N)=$ $P\left(1-\left(\frac{N}{\theta}\right)^{\alpha}\right)$ in a similar model. This extension reads (supposing $\mu=0$ )

$$
\begin{aligned}
& P^{\prime}=\varphi(P, N)-\left[r_{o}(N)+r_{i}(N)\right] P+r_{i}(N) N \\
& N^{\prime}=\varphi(P, N) .
\end{aligned}
$$

In the case of $\varphi(P, N)=P\left(1-\left(\frac{N}{\theta}\right)^{\alpha}\right)$, we easily see that Theorem 2 applies with $P=\Phi\left(N, N^{\prime}\right):=\frac{N^{\prime}}{1-\left(\frac{N}{\theta}\right)^{\alpha}}$, but one could imagine other examples, nonlinear in $P$, fitting into the framework of Theorem 2 as well.

Remark 5 The main results from Section 3 and Section 4 on the identifiability of the Gyllenberg-Webb model can be summarised as follows. For arbitrary functions (=observations) $P$ and $Q$, if the total tumour size $N=P+Q$ is strictly increasing with $N^{\prime}>0$,

- either there are no passage rates $r_{o}$ and $r_{i}$ yielding $P$ and $Q$,

- or $N$ satisfies a compatibility equation (with $\mu=0$ or $\mu>0$ ) and there are infinitely many passage rates $r_{o}$ and $r_{i}$ yielding $P$ and $Q$.

\section{Discussion}

The parameter identifiability problem gives useful insights in the potential of the Gyllenberg-Webb model. Under hypothesis $(\mathrm{H})$, introduced in Section 3, the model is able to reproduce any tumour growth model written as a firstorder ODE imposed on the total tumour size (cf. Corollary 1 ). If $f$ is concave, the passage rates can be chosen in a way that monotony $\left(r_{o}\right.$ nondecreasing, $r_{i}$ nonincreasing) holds. Consequently, the fact that Gyllenberg and Webb found passage rates corresponding to scalar ODEs used as tumour growth models is less a curiosity than an intrinsic property of their model in terms of flexibility. However, the non-uniqueness of corresponding parameters (cf. Figure 2) shows that only the associated function in the compatibility equation can be uniquely identified from the observations. If we want to identify the passage rates $r_{o}$ and $r_{i}$ from $P$ and $Q$, one of them has to be fixed in order to determine the other. Thus, the model leaves a latitude for the choice of parameters corresponding to the associated function. Moreover, by using the explicit form of the equivalence relation introduced in Definition 2, it has been shown that even a finite dimension parameter estimation problem with simple shapes of parametric functions can be ill-posed (cf. Corollary 2).

From a mathematical point of view, the particular form of the ODE system permits a uniqueness proof in a case of non-Lipschitz parameters, though admittedly in a limited setting. The regularity of the ODE on $N^{\prime}$ and the fact that $N$ is strictly increasing play a crucial role as they allow to break the problem down to a first-order ODE.

By posing $\mu=0$ in $(\mathrm{H})$, a biologically significant setting can be taken into account: Subcutaneous tumours often present a necrotic core trapped inside 
the tumour tissue, which thereby contributes to the total tumour volume. Obviously, considering $Q$ as the union of quiescent and dead cells introduces a systematic error in the model: The passage rate $Q r_{i}(N)$ should depend on quiescent cells only. This error could be overcome by introducing a third cell population $D$ of dead cells, thereby augmenting the number of parameters. However, SPECT imaging of proliferating cells does not permit to distinguish quiescent and dead cells (total tumour size is only extrapolated from the proliferating zone at the exterior). Therefore, $\mu=0$ is an acceptable compromise in terms of applicability.

The techniques used for establishing the parameter equivalence relation can be extended to a larger class of problems, including the Gyllenberg-Webb model with $\mu>0$ and a variant with a nonlinear birth rate of proliferating cells. In all cases, the increasing total tumour size is needed as a crucial assumption. The generalisation allows to transfer all relevant results. In a setting where most of the dead cells are eliminated by the immune system, the model with $\mu>0$ might be the most adequate one. One could also imagine that the birth rate $b P$ of proliferating cells could be insufficiently described by a linear model. Model choices can be founded on experimental validation, possibly in a murine model using SPECT imaging.

In the present work, it has been established that the non-identifiability of the passage rates is an intrinsic property in all of these models. For descriptive purposes, the ill-posedness of the parameter identification problem is not necessarily problematic. Any passage rate in a given equivalence class will describe not only global tumour growth (that is, $N$ ) in the same manner, but also both proliferating and quiescent cells. However, if the effect of a therapy is included in the model, the matter gets more delicate: The fact that quiescent cells become proliferating again when a therapy is applied clearly shows that the parameter equivalence cannot be true in this case, as $r_{i} \equiv 0$ would not include that effect but other passage rates in the same equivalence class, with a strictly positive function $r_{i}$, would. The real potential of the Gyllenberg-Webb model can therefore only be tested when confronting it with experimental data contrasting untreated and treated animals.

A pragmatic approach could exploit the non-identifiability of the passage rates: First, the equivalence class is identified using the model without treatment. Then, the data from the treated animals are used as an additional degree of freedom for model calibration when adding a therapy. Its descriptive performances can therefore be adapted to the quantity of data available.

Acknowledgements The author would like to thank Dr. T. Pourcher for providing the SPECT images and for his helpful comments on the usage of SPECT imaging in his laboratory. In addition to that, the remarks and propositions of Florence Hubert and Guillemette Chapuisat were of great value. Finally, the referees' feedback permitted to improve the quality and readability of this manuscript substantially. The author was partially supported by the Agence Nationale de la Recherche under grant ANR-09-BLAN-0217-01 and by the Cancéropôle PACA. 


\section{References}

(2012) WHO cancer fact sheet. http://www.who.int/mediacentre/ factsheets/fs297/en/

Alarcon T, Jensen HJ (2011) Quiescence: a mechanism for escaping the effects of drug on cell populations. J R Soc Interface 8:99-106

Amann H, Escher J (2006) Analysis II. Grundstudium Mathematik, Birkhäuser

Araujo RP, McElwain DL (2004) A history of the study of solid tumour growth: the contribution of mathematical modelling. Bull Math Biol 66:1039-1091

Brown D, Gatter K (2002) Ki67 protein: the immaculate deception? Histopathology 40:2-11

Coller HA (2011) The Essence of Quiescence. Science 334:1074

Coller HA, Sang L, Roberts JM (2006) A new description of cellular quiescence. PLoS Biol 4(3):e83

Colozza M, Azambuja E, Cardoso F, Sotiriou C, Larsimont D, Piccart M (2005) Proliferative markers as prognostic and predictive tools in early breast cancer: where are we now? Ann Oncol 16:1723-1739

D'Onofrio A, Fasano A, Monechi B (2011) A generalisation of Gompertz law compatible with the Gyllenberg-Webb theory for tumour growth. Math Biosci 230:45-54

Feller W (1939) On the logistic law of growth and its empirical verifications in biology. Acta Biotheoret 5:51-65

Franken PR, Guglielmi J, Vanhove C, Koulibaly M, Defrise M, Darcourt J, Pourcher T (2010) Distribution and dynamics of $(99 \mathrm{~m}) \mathrm{Tc}$-pertechnetate uptake in the thyroid and other organs assessed by single-photon emission computed tomography in living mice. Thyroid 20:519-526

Gardner L, Li Q, Park M, Flanagan W, Semenza G, Dang C (2001) Hypoxia Inhibits G1/S Transition through Regulation of p27 Expression. J Biol Chem 276(11):7919-7926

Gyllenberg M, Webb G (1989) Quiescence as an Explanation of Gompertzian Tumor Growth. Growth Develop Aging 53:25-33

Hahnfeldt P, Panigrahy D, Folkman J, Hlatky L (1999) Tumor development under angiogenic signaling: a dynamical theory of tumor growth,response and postvascular dormancy. Cancer Res 59:4770-4775

Hanahan D, Weinberg RA (2011) Hallmarks of cancer: the next generation. Cell 144:646-674

Hart D, Shochat E, Agur Z (1998) The growth law of primary breast cancer as inferred from mammography screening trials data. Br J Cancer 78:382-387

Hartung N (2011) Une étude de l'applicabilité du modèle de Gyllenberg et Webb. http://www.cmi.univ-mrs.fr/ hartung/memoire_master2_ niklas_hartung.pdf

Hittelman WN, Liao Y, Wang L, Milas L (2010) Are cancer stem cells radioresistant? Future Oncol 6:1563-1576

Iwata K, Kawasaki K, Shigesada N (2000) A Dynamical Model for the Growth and Size Distribution of Multiple Metastatic Tumors. J Theor Biol 203:177- 
186

Kozusko F, Bajzer Z (2003) Combining Gompertzian growth and cell population dynamics. Math Biosci 185:153-167

Kyle AH, Baker JH, Minchinton AI (2012) Targeting Quiescent Tumor Cells via Oxygen and IGF-I Supplementation. Cancer Res 72:801-809

Laird AK (1964) Dynamics of tumor growth. Br J Cancer 18:490-502

Liu J, Xia H, Kim M, Xu L, Li Y, Zhang L, Cai Y, Norberg HV, Zhang T, Furuya T, Jin M, Zhu Z, Wang H, Yu J, Li Y, Hao Y, Choi A, Ke H, Ma D, Yuan J (2011) Beclin1 controls the levels of p53 by regulating the deubiquitination activity of USP10 and USP13. Cell 147:223-234

Mellor HR, Ferguson DJ, Callaghan R (2005) A model of quiescent tumour microregions for evaluating multicellular resistance to chemotherapeutic drugs. Br J Cancer 93:302-309

Redmond KM, Wilson TR, Johnston PG, Longley DB (2008) Resistance mechanisms to cancer chemotherapy. Front Biosci 13:5138-5154

Ribba B, Watkin E, Tod M, Girard P, Grenier E, You B, Giraudo E, Freyer G (2011) A model of vascular tumour growth in mice combining longitudinal tumour size data with histological biomarkers. Eur J Cancer 47(3):479-490

Richard Fiardo P, Franken P, Lamit A, Marsault R, Guglielmi J, Cambien B, Graslin F, Lindenthal S, Darcourt J, Pourcher T, Vassaux G (2012) Normalisation to blood activity is a requirement for the accurate quantification of $\mathrm{Na} / \mathrm{I}$ symporter ectopic expression by SPECT/CT. PLoS ONE, accepted

Simeoni M, Magni P, Cammia C, De Nicolao G, Croci V, Pesenti E, Germani M, Poggesi I, Rocchetti M (2004) Predictive PharmacokineticPharmacodynamic Modeling of Tumor Growth Kinetics in Xenograft Models after Administration of Anticancer Agents. Cancer Res 64:1094-1101

Smallbone K, Gatenby RA, Maini PK (2008) Mathematical modelling of tumour acidity. J Theor Biol 255:106-112

Spinelli L, Torricelli A, Ubezio P, Basse B (2006) Modelling the balance between quiescence and cell death in normal and tumour cell populations. Math Biosci 202:349-370

Tredan O, Galmarini CM, Patel K, Tannock IF (2007) Drug resistance and the solid tumor microenvironment. J Natl Cancer Inst 99:1441-1454

Ubezio P, Cameron D (2008) Cell killing and resistance in pre-operative breast cancer chemotherapy. BMC Cancer 8:201

Wallbaum S, Grau N, Schmid A, Frick K, Neeb A, Sleeman JP (2009) Cell cycle quiescence can suppress transcription from an ecdysone receptor-based inducible promoter in mammalian cells. BioTechniques 46:433-440 